June 1986

\title{
Root Morphology Changes in Macaca Fascicularis Incident to Orthodontic Forces
}

Barry Rosenberg

Follow this and additional works at: https://opencommons.uconn.edu/sodm_masters

\section{Recommended Citation}

Rosenberg, Barry, "Root Morphology Changes in Macaca Fascicularis Incident to Orthodontic Forces" (1986). SoDM Masters Theses. 115.

https://opencommons.uconn.edu/sodm_masters/115 
ROOT MORPHOLOGY CHANGES IN MACACA FASCICULARIS

INCIDENT TO ORTHODONTIC FORCES

Submitted by: Dr. Barry Rosenberg

Advisors: Dr. Ravindra Nanda

Dr. Rohit Sachdeva

Dr. Alan Lurie 


\section{INTRODUCTION}

Changes in root surface morphology, such as root resorption as a result of orthodontic tooth movement, are recognized as an unfavorable possible consequence of orthodontic treatment. It is an irreversible change and its incidence is difficult to predict. Several risk factors have been implicated which increase individual predisposition: endocrine and metabolic disorders ${ }^{1-8}$, genetic ${ }^{9}$ and nutritional factors ${ }^{1,10-13}$; direction and tye of orthodontic tooth movement14-18; characteristics and degree of applied force systems 15,16,18-22; treatment duration 17,18,23,24; age of patient16,18,25-27; individual tooth type1,14,17,2125,26,28,29; and previous trauma to teeth ${ }^{16}$. There is a need to monitor cases in which risk factors tending towards root resorption are present.

The topography of root surfaces has been examined by different methodologies. The earliest reports by Ketcham 19-21 described changes seen radiographically after orthodontic treatment. Light and electron microscope examinations of changes in the periodontium following orthodontic tooth movement have been reported by Rygh30-33. Recently the scanning electron microscope has been used to this end17,34,35. The resorptive changes seen histologically are difficult to observe on conventional radiographs, but the radiographic changes could be demonstrated using a subtraction technique.

Subtraction radiography is a diagnostic technique used to detect small changes in tissue density. The standardization of films allows the visualization of small density changes which 
might otherwise be undetectable. The subtraction technique accentuates the slight changes in tissue density by optically removing unwanted, interferring and overlapping images 36. Subtraction radiography could be used to visualize, and thus monitor, early root surface changes in certain orthodontic cases with a predisposition toward root resorption.

A survey of the literature reveals that there are a numbr of papers which utilize techniques for observing teeth which have been subjected to tipping, extrusive and intrusive movements. However, there are no experiments which use an appliance designed to produce root movement and intrusion which places a high stress concentration at the root. In addition, subtraction radiography has not been utilized as a method to demonstrate changes in root morphology.

In the present investigation, orthodontic force was applied to the teeth of monkeys in order to induce changes in root surface morphology. The general objective of the study is to relate the changes observed clinically and radiographically to those occurring at the histologic level. The specific aims of this investigation are: 1. To observe the tissue reaction to an appliance with a defined force system designed to produce root movement and intrusion; 2. To correlate the change in spatial position of the tooth to the tissue reaction observed histologically; 3. To qualitatively describe the changes in root surface morphology using conventional and subtraction radiography. 


\section{LITERATURE REVIEW}

Although Schwarzkopf 37 described root resorption in 1887 using extracted teeth, little attention was paid to root resorption prior to Ketcham's $19-21$ reports in 1927 and 1929. Ketcham indicated that $22 \%$ of 224 patients and $21 \%$ of 500 patients whose teeth were examined radiographically after orthodontic treatment showed distinct evidence of root resorption. He compared these figures to the level of root resorption ( $1 \%$ ) he found in the population not subjected to orthodontic therapy. However, Ketcham reported only instances of obvious changes in root morphology and radiographic techniques were not standardized at that time, so that undistorted, reproducible films were not available.

other investigators have reported different incidences of root resorption using intra-oral radiographic techniques. In 1936, Rudolph25 reported that the incidence of root resorption was as high as $74 \%$ following orthodontic therapy. In 1940 , he 26 proposed that if treated with orthodontics long enough, every patient would experience some degree of root resorption.

Massler and Malone 28 attempted to improve the radiographic technique to examine root morphology. They examined each intraoral film using a binocular loupe with a $3 x$ magnification and looked at each tooth root from two different views. They claimed that these changes in methodology would allow the visualization of mild resorptive changes on the surface of roots. These investigators reported a considerable increase of root resorption in the apical area after orthodontic treatment. 
Phillips 29 studied 69 orthodontically treated patients and reported that over $80 \%$ of maxillary incisors exhibited root resorption after treatment. In a similar study, DeShields 24 found a significant correlation between apical tooth movement and aplcal resorption. His findings were based on lateral cephalometric headplates of 52 treated class II, division I malocclusions.

Dellinger ${ }^{38}$, in a study on changes following intrusion in monkeys, stated that optimal intrusion could be achieved with 50 grams of force. He also found that root resorption did not appear to be related to the distance that teeth were intruded. Williams 39 , in a cross-sectional study, induced root resorption in rats by means of orthodontic forces and described the ensuing histological changes in their chronologic sequence. He observed the appearance of resorptive activity along the root surface at eisht days.

Reitan 18 , in an extensive study using human subjects, denonstrated that apical root resorption is a complex process inrolving appositional and resorptive changes. He found that apical root resorption tends to start adjacent to a hyalinized zone and is more likely to occur in cases where the periodontal Iigament compression is strong and of long duration. He observed that root resorption occurredin the majority of cases of tooth movement, even with light forces, and increased in cases in which strong forces had been applied. Reitan demonstrated that the presence of a cementoid layer on the root delays the resorptive process. 
enhanced visual perspective. In 1972, Kvams4,Jb published SEM studies of changes in the cementum of human teeth following orthodontic tooth movement. He found small root resorptions in the pressure side in all experimental premolars after ten days. However, his studies were restricted to a description of the cervical and mid-root pressure regions and no mention was made of any apical change.

Harry and Sims ${ }^{17}$ investigated the topography of human root resorption under continuous intrusive orthodontic force application of varying magnitude and duration. They observed loss of cementum and dentin after fourteen days and a noticeable decrease in root length after thirty-five days with fifty grams of force. The amount of resorption increased markedly with the duration of the force and to a lesser extent with the magnitude of the appliance activation.

Subtraction radiography offers a method for improving the detection of lesions which are small and difficult to perceive visually. The rationale is based on the fact that unchanged anatomical structures cancel in the subtraction image 41 . This results in a less complex picture; thus allowing for the discrimination of small morphologic changes. The success of this technique is contingent on the cancellation of superimposed structures such as cranial anatomy obscurring opacified blood vessels. The rigidity of the teeth and supporting bone make it 
possible to obtain reproducible projections with the aid of an occlusal template42. In dental radiography, the process of subtraction has been used in diagnosing periapical 43 and periodontal $36,42,44,45$ bone lesions, and in longitudinal cephalometric growth studies 46 . 


\section{MATERIALS AND METHODS}

The present study utilizes three Macaca fascicularis monkeys over a seven week experimental period. The monkeys had been used in a previous study in which cranial implants were placed in both jaws to study the maxilla. The monkeys selected had all the permanent teeth erupted except the third molars. All monkeys were female. No separate control monkeys were used. The lower left cuspid was used for the experimental procedure and the lower right cuspid served as the control in each animal.

The monkeys were housed in the animal care section of the UCONN Health Center in specially designed cages. The temperature, humidity and light were controlled. A specially prepared soft diet was fed to them and fresh fruits were given as a supplement. Water was available ad libitum.

\section{Anesthesia}

For procedures of less than 30 minutes duration, ketamine hydrochloride $(15 \mathrm{mg} / \mathrm{kg})$ was administered intramuscularly. When periods of longer or deeper sedation were required, acepromazine $(7 \mathrm{mg} / \mathrm{kg})$ was also administered intramuscularly with atropine ( $7 \mathrm{mg} / \mathrm{kg})$ to block the bradycardia associated with acepromazine.

\section{Orthodontic Procedures}

A mandibular alginate impression was taken on the animals and poured in orthodontic plaster in order to fabricate an acrylic crown to fit over the posterior teeth. The posterior teeth were "ditched" and right and left multi-tooth acrylic crowns were processed with cold cure acrylic. The acrylic bands 
were trimmed, polished and bonded to the posterior teeth. The bonding procedures were carried out with system $1^{*}$. On the left side a .022 x .028 non-torqued edgewise bracket was bonded at the first molar area on the band and on the cuspid. All brackets were placed parallel to the occlusal plane.

Amalgam reference points were placed in both the right and left lower cuspids to aid in the superimposition of the radiographs. The placement at the incisal edge was important because it had to be at a distance from the bracket.

In the experimental side, a root spring made of $.018 \times .025$ stainless steel was ligature-tied from the posterior bracket to the cuspid bracket. The appliance induced orthodontic intrusion as well as distal root movement of the cuspid. The appliance delivered a high moment to force ratio to the cuspid and by increasing the pre-activation bends on the distal segment with respect to the cuspid, intrusion of the cuspid occurred.47(Fig. 1) The root spring was reactivated at three weeks and delivered a large moment to the experimental teeth. The force system of the spring was calibrated prior to insertion. The actual force and moment values used are given in Table $I$.

\section{Table I}

Monkey

1

2

3

*ormco/sybron, Glendora, CA 91740
Distance

$\begin{array}{cl}\text { Ant. } & 125 \mathrm{~g} \\ \text { Post. } & 200 \mathrm{~g}\end{array}$

Ant. $175 \mathrm{~g}$

Post. $250 \mathrm{~g}$

Ant. $150 \mathrm{~g}$

post. $200 \mathrm{~g}$
$24 \mathrm{~m} \mathrm{~m}$

Moment

$3000 \mathrm{~g}-\mathrm{mm}$

$4800 \mathrm{~g}-\mathrm{mm}$

$16 \mathrm{~m} \mathrm{~m}$

$20 \mathrm{~m} \mathrm{~m}$
$2800 \mathrm{~g}-\mathrm{mm}$

$4000 \mathrm{~g}-\mathrm{mm}$

$3000 \mathrm{~g}-\mathrm{mm}$

$4000 \mathrm{~g}-\mathrm{mm}$ 
On a weekly schedule, the monkeys were anesthetized and the tooth movement and appliance were more closely inspected. Any loose bands, broken wires or bent appliances were replaced and an oral prophylaxis was performed.

\section{Radiologic Methods}

Both baseline and post-treatment films must be taken at the same voltage, current and time parameters. Object-film distance, and horizontal and vertical angulations must be identical from one film to the next. The fabrication of a standardized device for taking reproducible dental radiographs has been described ${ }^{36}$. A child anterior precision instrument device was used for film positioning (Fig. 2). The device was prepared with the following modification: Instead of superimposing on a quadrant of teeth, the template was made solely for the cuspid. Deep grooves were cut in the cuspid with a crown and bridge bur and a single tooth impression was made using cold-cure acrylic. This impression was trimmed and bonded to an acrylic plate sized $8 x$ $13 \mathrm{x} 32 \mathrm{~mm}$. This piece was fastened to the precision instrument with two \# 8 bolts with nuts.

The $x$-ray machine used was a siemens Heliodont dental unit with a long cone at settings of $90 \mathrm{kVp}$, and .64 second exposure. Kodak size 0 , D-speed film was used for all exposures. Periapical films were processed by hand developers using FR* developing and fixing chemistry. Step-wedge films were taken at each session to monitor machine and chemistry performance. The

Masel Co, Philadelphia, PA, 19154

FR chemicals, Cine Magnetics Inc., Yonkers, NY 10703 
radiographs were examined visually to see if any changes in root morphology could be detected. For each monkey the baseline and post-treatment radiographs were also analyzed with a subtraction radiographic technique.

\section{Subtraction Technique}

The pre-treatment periapical radiograph was used to make a masque film using Kodak masque film on a Cronex* printer. The time settings were varied to produce optimum density. Superimpositions of the masque film on the post-treatment radiograph was performed using a dissecting microscope at $10 x$ with a high intensity lamp projected from behind the film. The root canal and the crown of the cuspid were used for superimposing the films. The print was made using Kodak print film on a cronex printer. Again, the time settings were set to produce optimum density.

\section{Sacrifice of Animals}

The animals were sacrificed after seven weeks by injecting a fatal dose of ketamine hydrochloride directly into the heart of each animal. Following this, the mandible was removed and hemisected with aid of an electric bone saw.

\section{Histologic Preparation}

The two halves of the mandible were immediately fixed in $10 \%$ formalin and the tissues were then decalcified in $5 \%$ formic acid. After decalcification, all excess tissue was removed. This procedure allowed for better control of the tissue block. **** DuPont, St. Louis, MO 63103 
Longitudinal serial sections 7 um thick were cut parallel to the mid-sagittal plane. All sections were stained with hematoxylin and eosin.

Data Interpretation

All slides were examined for root resorption as well as bony changes and periodontal ligament damage. The orientation of the trabeculae and PDL were observed for any further indication of the direction of tooth movement. The radiographic data collected are a qualitative assessment of the observable changes. Conventional and subtraction radiography were compared with respect to their ability to detect small changes in radiographic appearance due to changes in tissue density. An attempt was made to correlate the observed radiographic changes with the histologic appearance of the cuspid. 


\section{RESULTS}

\section{Visual Examination}

All bands, brackets and wires stayed in placed for the duration of the experiment. The action of the spring on the experimental teeth induced observable intrusion, tipping and distal root movement of the cuspid (Fig. 3 ). Extrusion and mesial root movement of the buccal segment were observed (Fig 4). The amalgam implants did not remain as placed in the cuspid teeth and therefore could not be used for superimposition of the subtraction films.

\section{Histologic Findings}

Histologically the control teeth and their supporting structures appeared normal. The periodontal ligament (PDL) was approximately constant in width (Figs. 5 \& 6). Tooth resorption, confined to the cementum, was noticed at some of the cuspid apices. Observation of the bone revealed normal trabeculae with no areas of overtly increased bone resorption or apposition ( Fig. 7).

The cuspids of the experimental group had numerous resorptive areas that were confined to the middle and apical onethird of the distal aspect of the root and at the apex. The resorptive activity at the apical third of the distal aspect was comparatively most predominant. Resorptive areas were also noted at the apex of the posterior anchorage teeth. The resorptive areas were in typical erosive lacunae and ranged from small lesions barely through cementum to expansive, coalescing resorptive areas that were deep into the underlying dentin. Once 
the resorptive activity reached the dentin, it appeared to spread out. The resorptive lesions typically contained a combination of active and inactive areas. A reversal Iine could be seen separating the odontoclasts from the newly formed repair tissue (Figs. 8 \& 9).

odontoclasts with multiple nuclei were observed at the resorbing site although demarcation of the cell boundaries could not be clearly delineated. Their morphology and staining pattern were variable; however, in general the cytoplasm was eosinophilic and stained lightly. The nuclear staining was more variable ranging from lightly stained to darkly stained with distinct nucleoli.

Two areas of cementum apposition were observed. First, cementum was laid down on the mesial aspect of the cuspid root. In the second, a hypercementosis was seen at the apex of a second molar. The cementum in non-resorbed areas was of normal morphology.

The PDL surrounding the experimental teeth had a rich cellular content with an abundance of fibroblasts with basophilic cytoplasm. The PDL appeared edematous and the fibers were in a disorganized arrangement. There appeared to be an increased amount of new capillary budding and a hyperemic state compared to control teeth (Fig. 10). There were no areas of hyalinization.

observation of the alveolar bone revealed areas of bone formation in the posterior segment with the trabecular direction corresponding to the line of force application and immature fibrous bone surrounding them. 


\section{Radiographs}

All radiographs were exposed and processed at identical voltage, current, time and temperature parameters and processed with the same concentration of chemical solutions. Masque films and subtraction films were processed to ideal contrast by varying the exposure times. It was attempted to have identical projection angles and film distances for all radiogaps. However, due to the small size of the monkey's oral cavity, ideal placement of both before and after films could not always be achieved.

Viewing the pre- and post-treatment radiographs by conventional means revealed no evidence of changes in root morphology (Figs.11 \& 12). A slightly widened PDL space and thickened lamina dura on the distal aspect of the experimental root could be detected. In some films, a clear image of the cuspid root was obscured by metal implants (placed for another experiment) or normal anatomy such as adjacent roots or the mylohyoid ridge. This problem could not be avoided because ideal film placement was not always possible.

Subtraction of the films was performed by superimposing to best fit using the coronal and root canal anatomy. In both control and experimental teeth, no clear evidence of root morphology changes could be detected. A dark zone was observed at the apex of both the control and experimental cuspids. This was probably due to efforts in superpositioning (Fig. 13 \& 14). 


\section{DISCUSSION}

The present study was undertaken to observe the radiographic and histologic changes produced from the use of a defined force system exerting root movement and intrusive forces. The mandibular left cuspid of Mascaca fascicularis monkeys was used as the experimental tooth. The forces were in the clinical range for human subjects49. Periapical radiographs were taken and viewed conventionally and with a subtraction technique. The force system produced the desired tooth movement as was shown by visual, radiographic and histologic examination.

Reitan ${ }^{18}$ has stated that the magnitude of the applied force is the most critical causative factor in the onset of root resorption. Dellinger ${ }^{8}$, in a study on primates, reported that fifty grams was the optimum force level for intrusion of premolars. He selected this value because he observed no root resorption. In the present study, moment values of 2800 to 3000 8-mm were chosen. These values are comparable to moment values proposed by Burstone 49 for cuspid root movement in humans. Since the primate cuspid has a smaller root surface area than the human, a greater stress should be felt by the primate root. In the present study, a radiographic and histologic examination revealed no root resorption.

The histologic findings of this study support previous observations by Reitan ${ }^{18}$ and Rygh40 that root resorption also occurs when the forces are of a long duration. Kvam34 has reported that dentinal resorption will occur at 25 days in humans 
with a force of $50 \mathrm{grams}$. In the present study, surface cemental and dentinal resorptions were seen after the experimental period of 49 days.

The findings of this study are in agreement with those of Kvam34,35 that minor root resorption occurs on most teeth subjected to orthodontic movement. The resorptions observed were more numerous on the distal aspect of the root, which is the surface undergoing compression. On the other hand, the surfaces placed in tension reacted with slight cementum deposition. This is in agreement with the findings of Rygh 30,31 . It is important to note that these observations were made immediately after the orthodontic movement has ended. Thus, healing of the root surfaces had not been able to take place. Overall the cemental surface shows remarkable ability to heal following small resorption areas. This is contrary to apical root resorption which in effect causes shortening of the roots and is irreversible. 18

Surface root resorptions that were mostly limited to the cementum were noted in all experimentally moved teeth. These resorption tend to spread out once they reach the dentin 23 . In addition, the rapid formation of extensive resorptive defects has been purported to take place due to the thin walls separating the individual lacunae ${ }^{4}$. In young individuals, the root surfaces are normally covered by a precementum 48 and it has been shown that such tissue resists resorption more readily than cementum ${ }^{18}$. The presence of the precementum may have prevented apical root 
resorption during the observation period. Apical root resorption may have occurred had the experimental period been extended or with the use of a higher magnitude of force.

The changes observed histologically could not be conclusively correlated with the radiographic picture. This was probably because the resolution of the radiographs is not sufficient to see such small changes in root morphology. The changes observed radiographically were widening of the PDL space and thickening of the lamina dura. In dental radiography only the most extensive resorptions are detectable. Since no apical blunting of the teeth was observed histologically, there were no obvious changes detectable radiographically.

The subtraction technique could be used if larger animals or smaller films are used to allow to ideal film placement. Once better superimposition of the films is achieved, computer digitization could be adapted to the subtraction technique to allow precise quantification of the changes observed. This technique as used in the study, did not help correlate histological changes seen at the cemental surface to those at the radiographic image level.

The amalgams placed for radiographic superimpositioning were not retained in the cuspids. The small size of the clinical crowns made it difficult to place a bonded bracket and an amalgam for superimposition. Crowning the cuspids with a cast bracket would alleviate the need to place an amalgam because the crown could be used for superimposition. In addition, the crown could be prepared with a precision attachment to allow rigid and stable placement of the film holder. 
The present investigation is a pilot study evaluating the ability to monitor early root morphology changes. The next step in this line of research would be to establish the sensitivity of the subtraction technique using dried skulls. one could introduce measured artificial lesions to teeth and attempt to observe their presence with conventional and subtraction radiography. Subtraction radiography may be able to detect changes in root morphology that cannot be observed with conventional radiography. This may play as an important clinical tool in determining force levels for various types of tooth movements as well as for individuals with compromised periodontal health. 


\section{SUMMARY AND CONCLUSIONS}

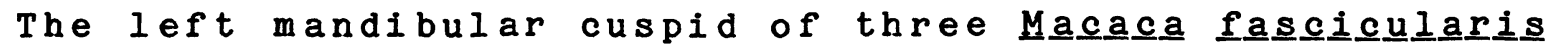
monkeys was subjected to intrusion and root movement for 49 days. The contralateral tooth served as a control. The force was calibrated to be within the clinical range for human root movement. Standardized periapical radiographs were taken and viewed conventionally and with a subtraction technique.

The results of this study show that the force system used caused no apical root resorption as noted by blunting or shortening of roots. However, surface root resorptions were noted at several sites on roots of experimentally moved teeth. The resorption was limited to cementum in most areas but once it reached dentin it tended to spread out. The changes observed histologically could not be conclusively correlated with the radiographic appearance due to the problems associated with superimposition and resolution of the radiographs. 


\section{FIGORE 1}

A

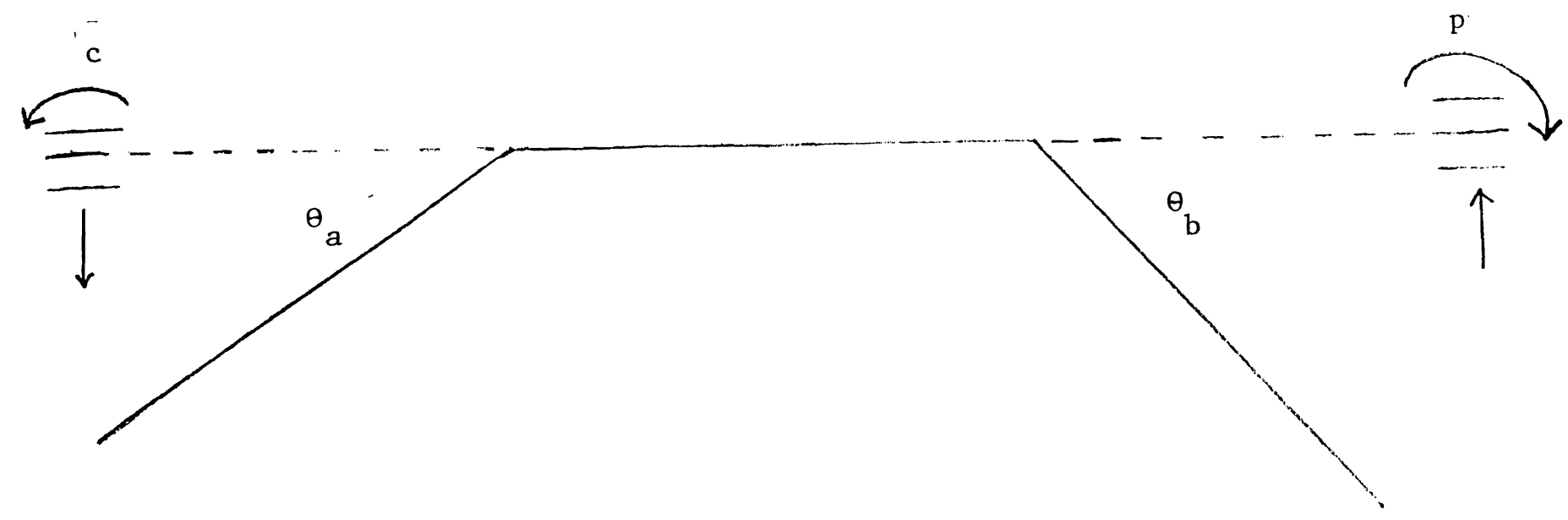

Orthodontic appliance producing distal root movement of the cuspid (c). Notice the differential pre-activation bends with the posterior being greater; that is $O_{b}$ is greater that $0_{a}$. Vertical forces are created with an intrusive component being felt by the cuspid, and an extrusive force by the posterior segment (p). 


\section{FIGURE 2}
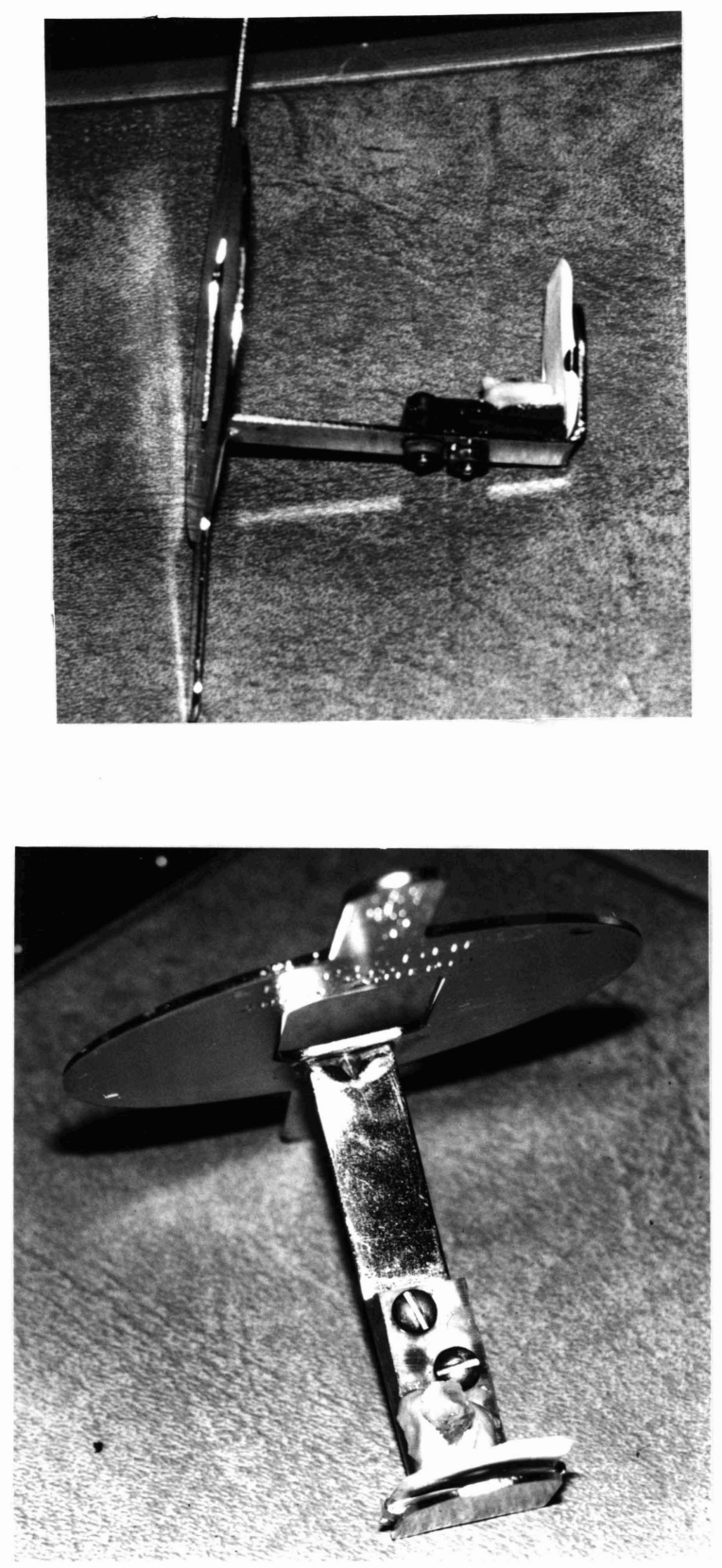

Two views of the modified precision instrument device used to position film for periapical radiograph. 


\section{FIGURE $3 \mathbf{a}$}

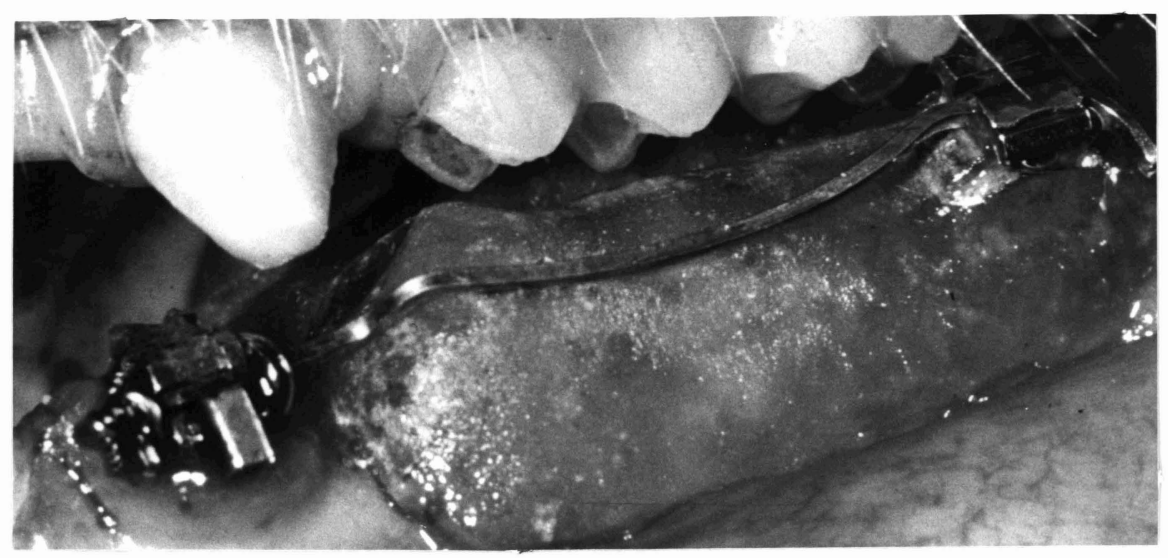

Experimental cuspid with wire to induce root movement and intrusion.

FIGURE 3b

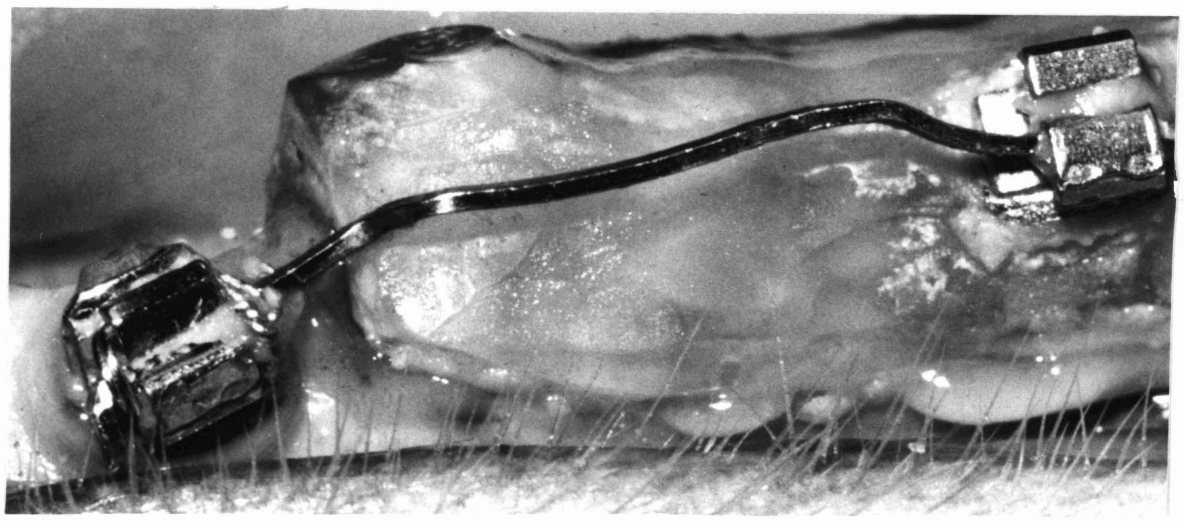

Experimental cuspid after tooth movement. 


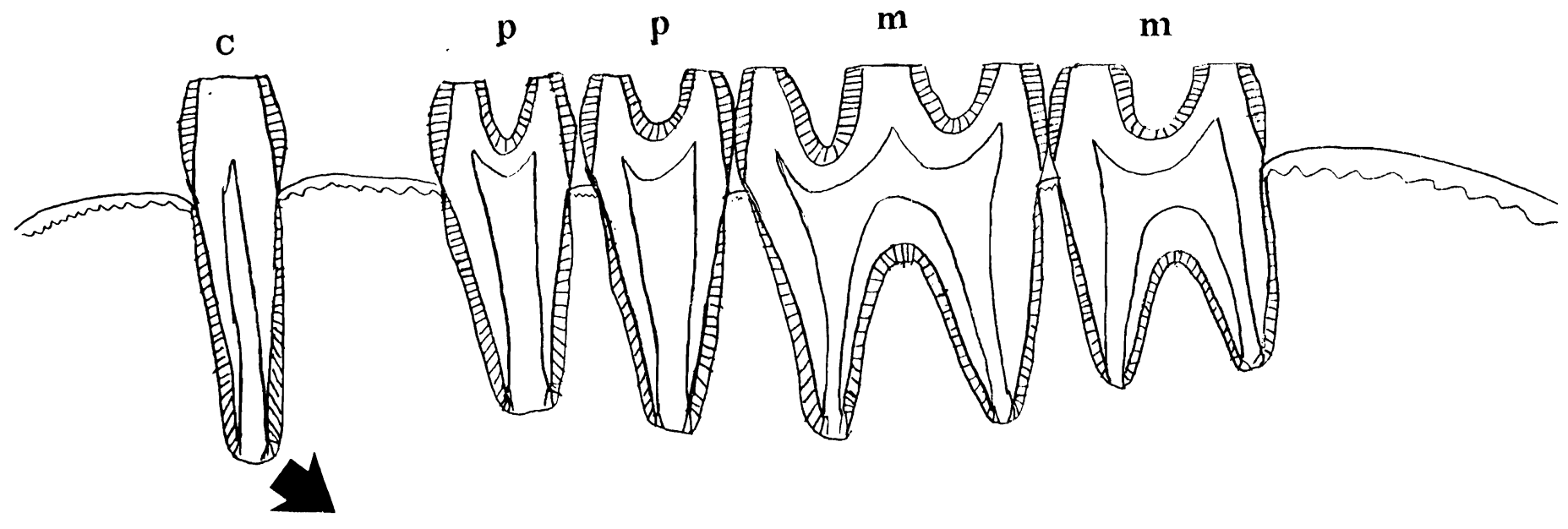

Drawing of monkey mandible indicating direction of cuspid (c) movement. Posterior anchorage teeth are comprised of two premolars (p) and two molars (m). 


\section{FIGURE 5}

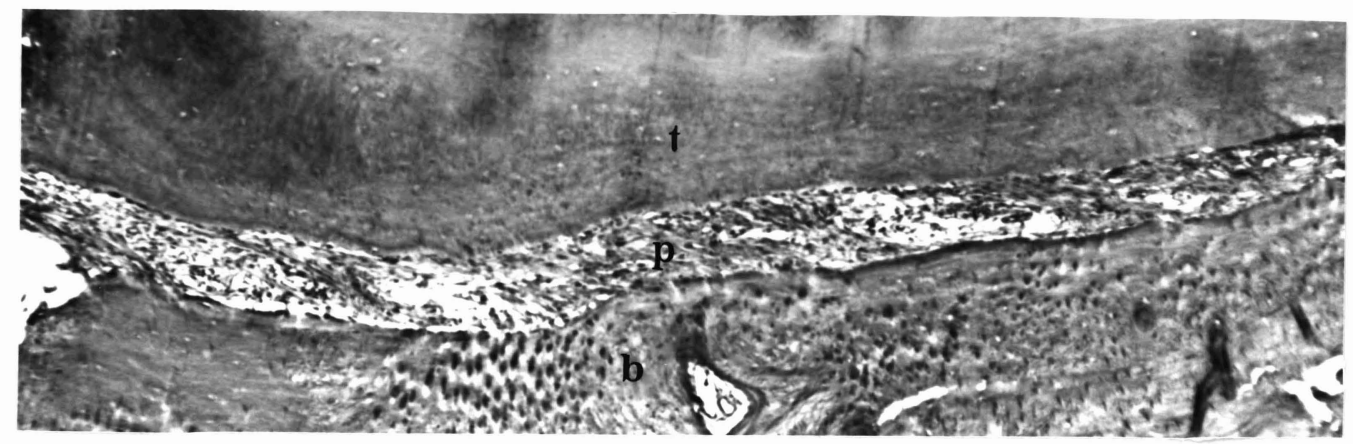

Control animal, apical area of cuspid. Low power view demonstrating normal relationship between the cuspid ( $t$ ), periodontal ligament ( $p$ ), and alveolar bone (b). H \& E $125 x$.

\section{FIGURE 6}

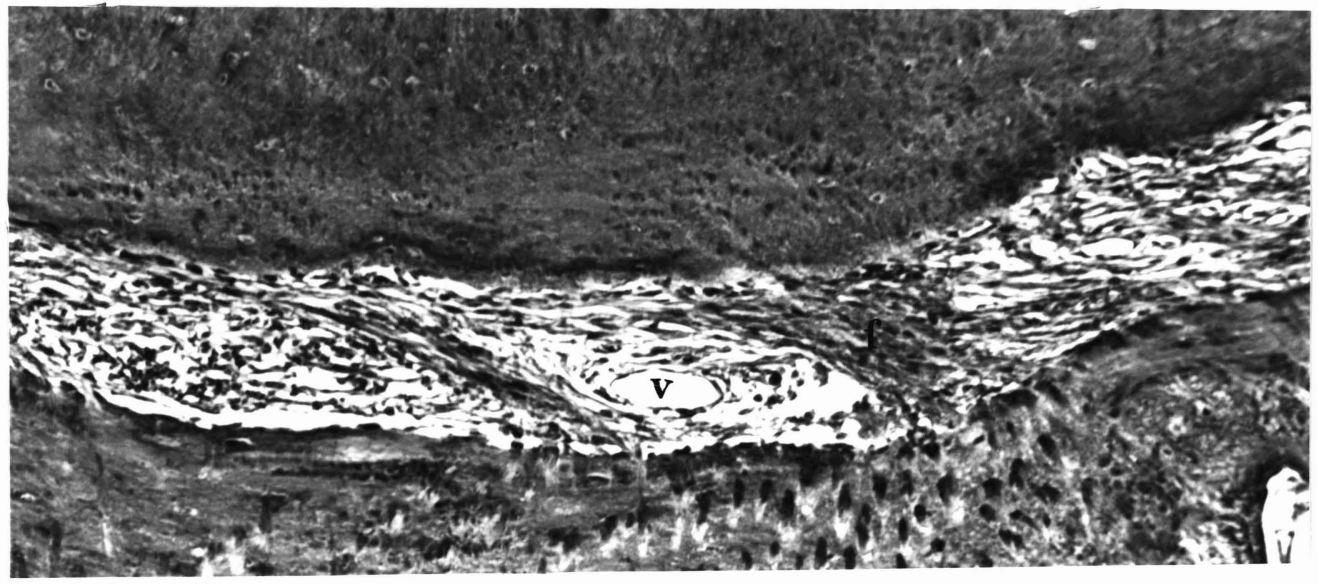

High power view of same control animal (Fig. 1) demonstrating normal periodontal relationship. Note transverse fibers (f) and blood vessels (v). H \& E $250 x$. 


\section{FIGORE 7}

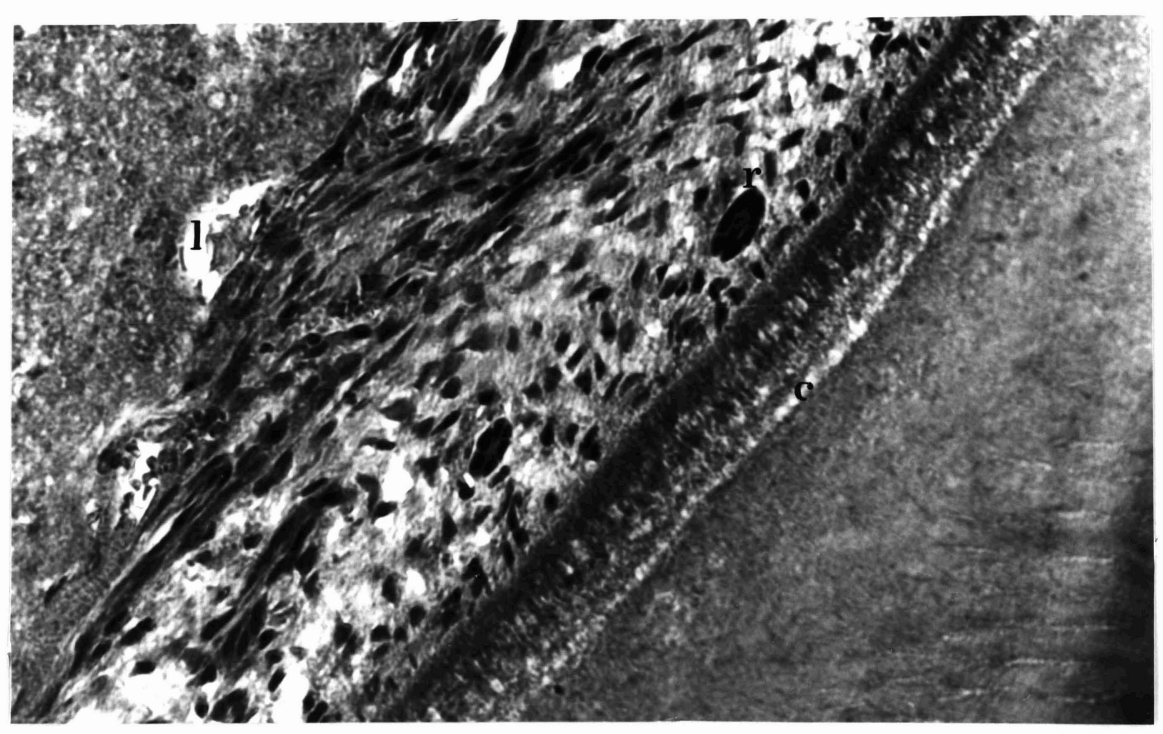

Control animal--physiologic bone resorption, mid-root area of cuspid. Note small lacunae (1) on bone surface in contrast to intact, uniform surface of cementum (c). Note rests of Malassez ( $r$ ) in PDL. H \& E $400 x$. 


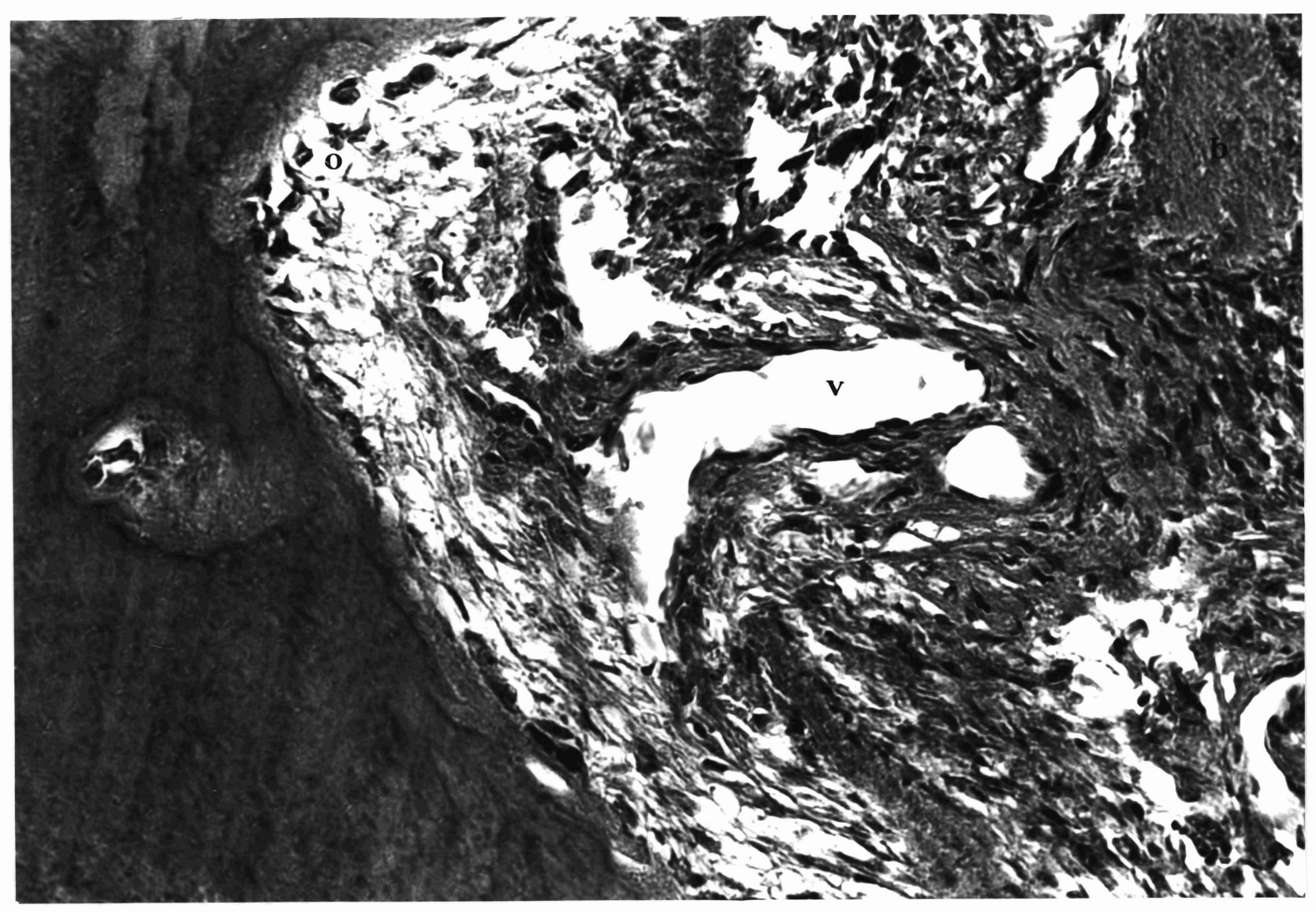

Experimental animal--external root resorption at 7 weeks. Abundant odontoclasts (0) are present on irregular surface of resorption lacunae. Vascular channels (v) within stretched periodontal ligament are widely dilated and fibers reaching to bone (b) are in disarray. H \& E $450 x$. 


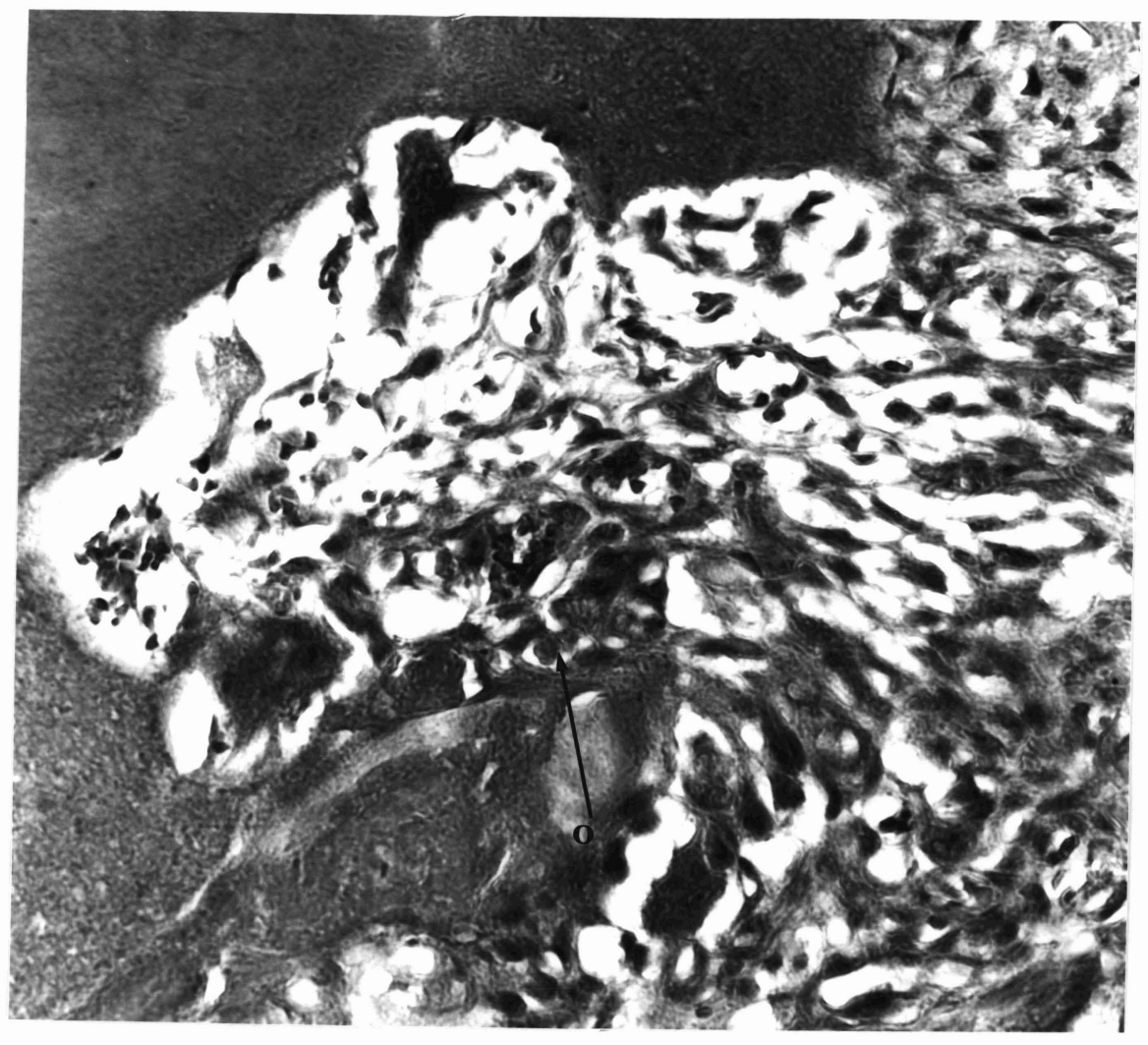

High power view of resorption lacunae in experimental animal at 7 weeks. Note marked irregularity of root surface containing multinucleated odontoclasts (o). H \& E 550x. 


\section{FIGURE 10}

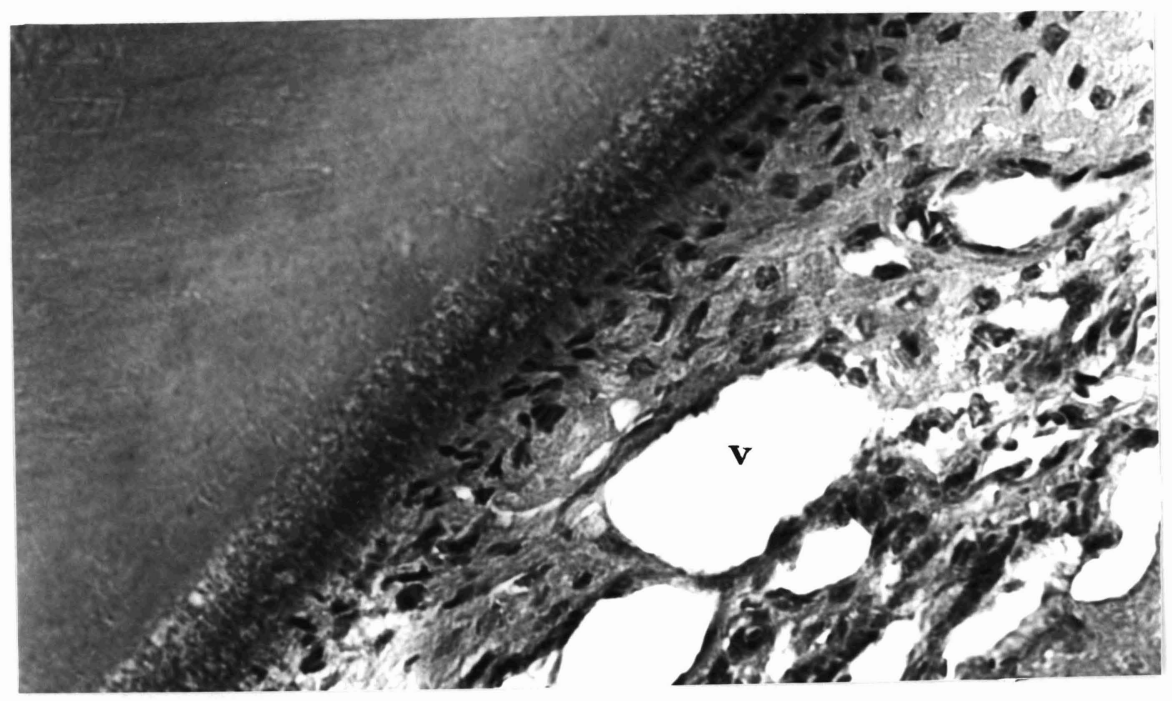

Periodontium of experimental animal at 7 weeks. Periodontal ligament appears edematous and there is marked vascular dilitation ( $v$ ). Note the lack of discrete periodontal collagen fibers. H \& E $250 x$. 


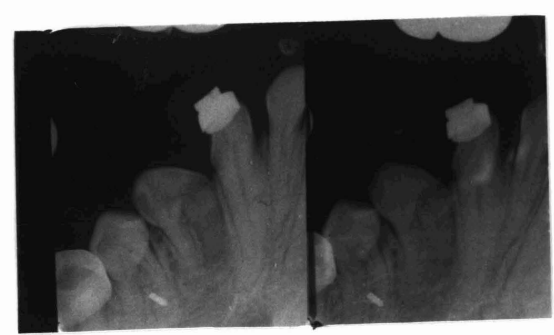

a 1

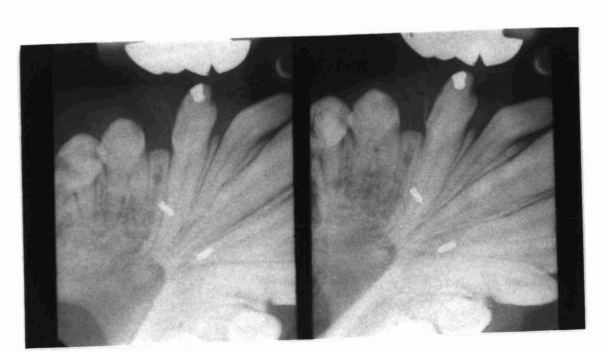

b
2

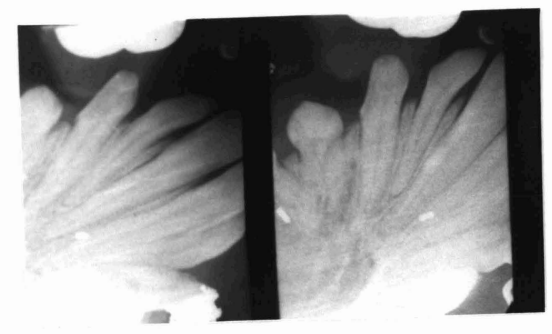

2

Standardized periapical radiographs of control teeth of 3 animals (a-c), before (1) and after (2) treatment.

FIGURE 12
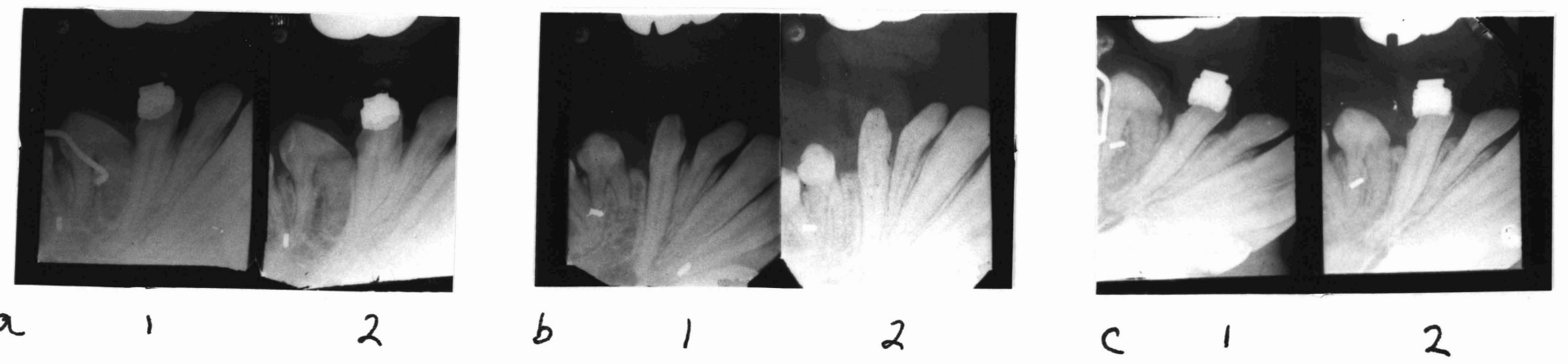

Standarized periapical radiographs of experimental teeth of 3 animals $(a-c)$, before (1) and after (2) treatment. 


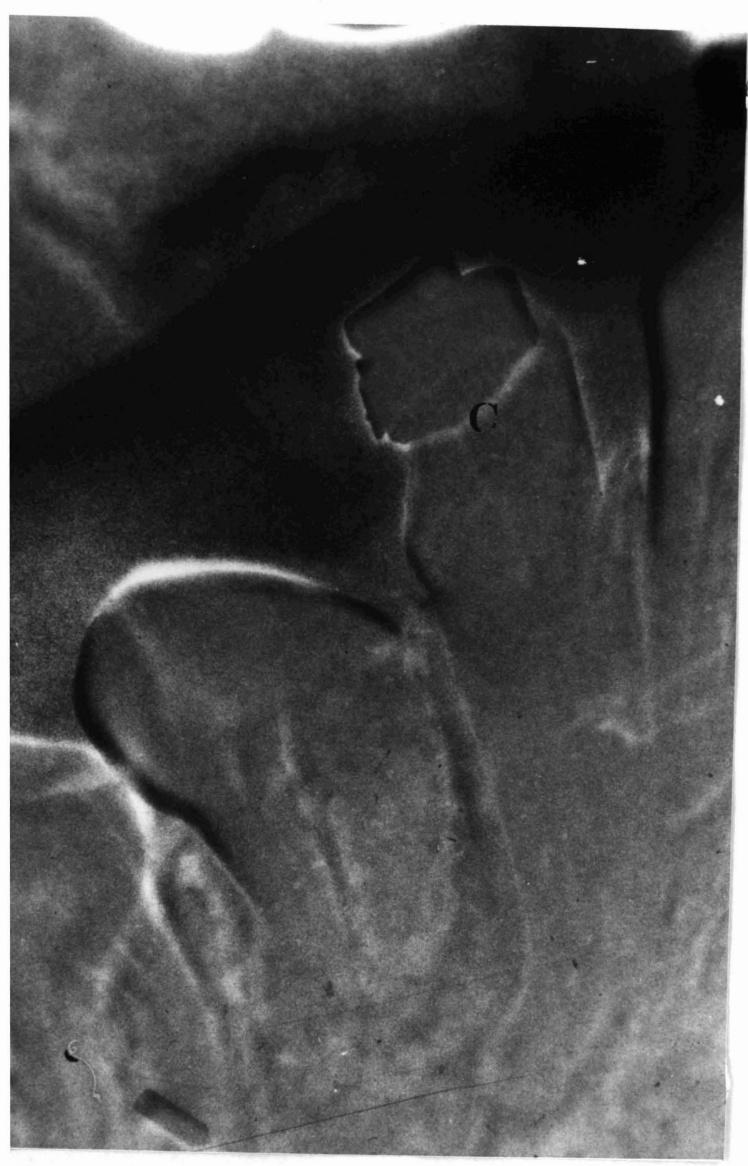

FIGURE 13
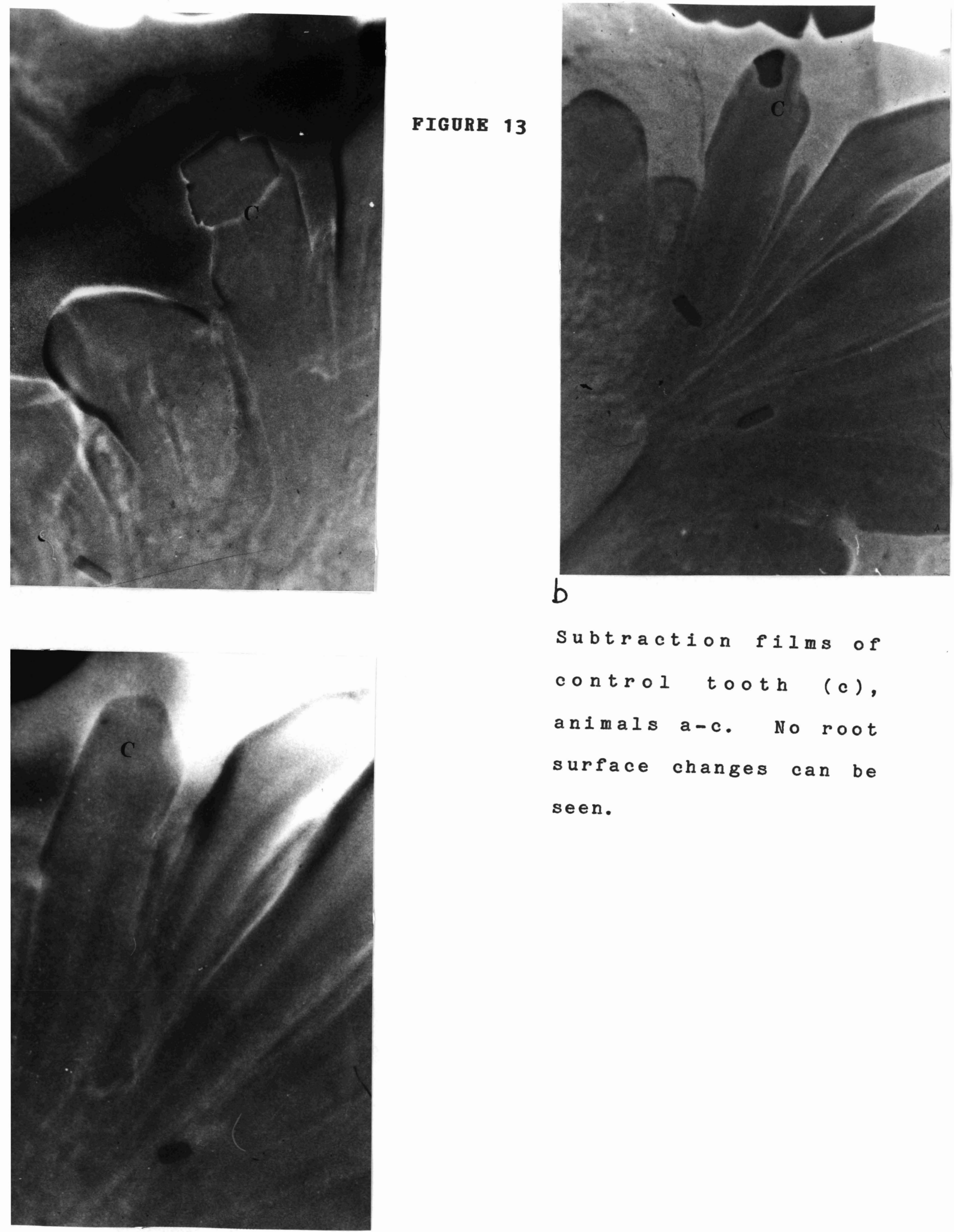

Subtraction films of control tooth (c), animals a-c. No root surface changes can be seen. 


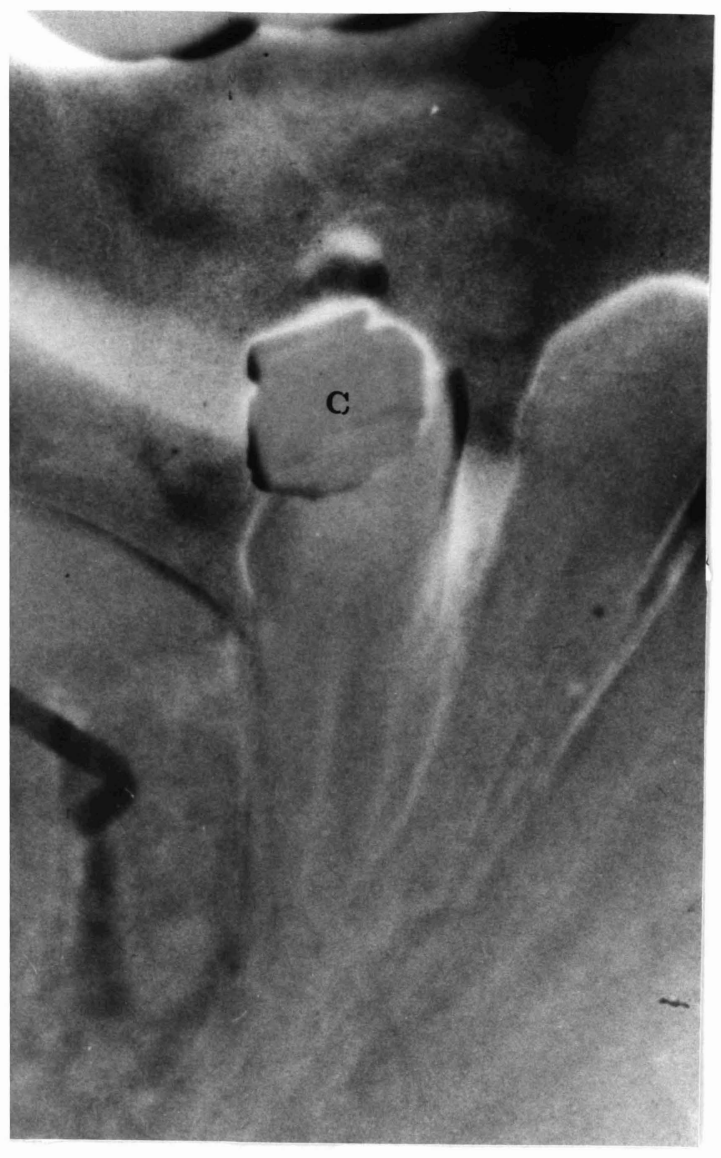

FIGURE 14

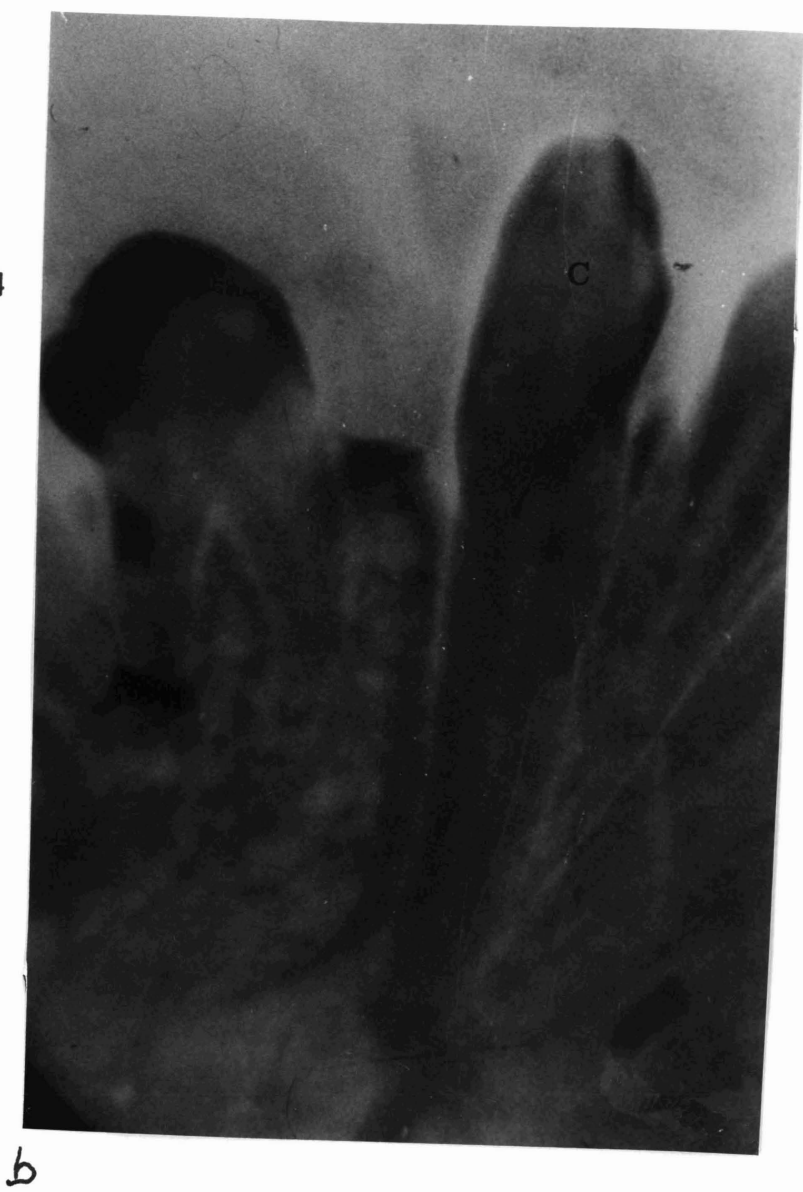

Subtraction films of experimental tooth (c), animals a-c. Due to inadequate superimposition and the resolution of the subtraction technique, root surface changes cannot conclusively be seen. 


\section{References}

1. Becks, H. Root resorptions and their relation to pathologic bone formation. Int. J. Orthod. 22:445-82, 1936.

2. Becks, H. Orthodontic prognosis: evaluation of routine dentomedical examinations to determine "good and bad risks." Am. J. Orthod. 25:610-24, 1939 .

3. Becks, H. and Cowden, R.C. Root resorptions and their relation to pathologic bone formation. Part II. Am. J. Orthod. 28:513-26., 1942 .

4. Carman, J. Arrested root absorption during orthodontic treatment. Int. J. Orthod. 23:35-7, 1937 .

5. Cross, S. Endocrines: Their relation to orthodontic diagnosis and treatment planning. Am. J. Orthod. 418-40, 1948 .

6. Tager, N. Endocrine problems in orthodontics. Am. J. Orthod. $37: 867-75,1951$.

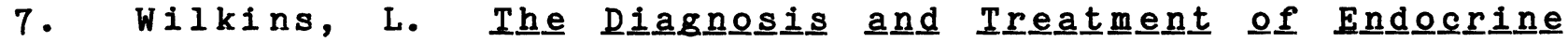

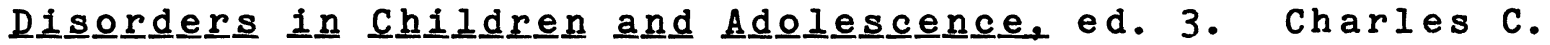
Thomas Publishers, Springfield, Ill, 1965.

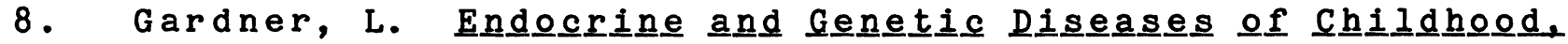
W.B. Saunders Company, Philadelphia, 1969.

9. Newman, W.G. Possible etiologic factors in external root resorption. Am. J. Orthod. 67:522-39, 1975.

10. Marshall, J.A. Studies on apical absorption of permanent teeth. Part I. Int. J. Orthod. 16:1-19,1930.

11. Marshall, J.A. The relation of malnutrition to dental pathology. Int. J. Orthod. 17:527-51,1931.

12. Marshall, J.A. Deficient diets and experimental malocclusion considered from the clinical aspect. Int. J. Orthod. $18: 438-49,1932$.

13. Marshall, J.A. The classification, etiology, diagnosis, prognosis, and treatment of radical resorption of teeth. Int. J. Orthod. 20:731-49, 1934 .

14. Hemley, S. The incidence of root resorption of vital permanent teeth. J. Dent. Res. 20:133-141, 1941.

15. Stuteville, 0.H. Injuries caused by orthodontic forces and the ultimate results of these injuries. Amer. J. Orthod. and Oral Surg. 24:103-18, 1938 . 
16. Linge, B. O. and Linge, L. Apical root resorption in upper anterior teeth. Europ. J. Orthod. 5:173-83, 1983.

17. Harry, M.R. and Sims, M.R. Root resorption in bicuspid intrusion. A scanning electron microscope study. Angle Orthod. $52: 235-58,1982$.

18. Reitan, K. Initial tissue behavior during apical root resorption. Am. J. Orthod. 44:68-82, 1974 .

19. Ketcham, A.H. A preliminary report of an investigation of apical resorption of permanent teeth. Int. J. orthod. $13: 97-127,1927$.

20. Ketcham, A.H. A radiographic study of orthodontic tooth movement: A preliminary report. J.A.D.A. 14:1557-1596, 1927 .

21. Ketcham, A.H. A progress report of an investigation of apical root resorption of vital permanent teeth. Int. J. Orthod. and Oral surg. 15:310-28, 1929.

22. Schwarz, A.M. Tissue changes incident to tooth movement. Int. J. Orthod. and Oral Surg. 18:331-52, 1932 .

23. Stenvik, A. and Mjor, I.A. Pulp and dentine reactions to experimental tooth intrusion. A histologic study of the initial changes. Am. J. Orthod. 57:370-85, 1970 .

24. DeShields, R.W. A study of root resorption in treated class II, division 1 malocclusions. Angle orthod. 39:231-45, 1969 .

25. Rudolph, C.E. A comparative study in root resorption of tooth resorption in permanent teeth. J.A.D.A. 23:822-825, 1936 .

26. Rudolph, C.E. An evaluation of root resorption occurring during orthodontic treatment. J. Dent. Res. 19:367-71, 1940 .

27. Rosenberg, H.N. An evaluation of the incidence and amount of apical root resorption and dilaceration occurring in orthodontically treated teeth having incompletely formed roots at the beginning of Begg treatment. Am. J. Orthod. $61: 524-5,1972$.

28. Massler, M. and Malone, A.J. Root resorption in human permanent teeth. A roentgenographic study. Am. J. Orthod. 40:619-33, 1954 .

29. Phillips, J.R. Apical root resorption under orthodontic therapy. Angle Orthod. 25:1-22, 1955. 
30. Rygh, P. Ultrastructural vascular changes in pressure zones of rat molar periodontium incident to orthodontic tooth movement. Scand. J. Dent. Res. 80:307-321, 1972 .

31. Rygh, P. Ultrastructural cellular reactions in pressure zones of rat molar periodontium incident to orthodontic tooth movement. Acta 0dont. Scand. 30:575-93, 1972.

32. Rygh, P. Ultrastructural changes of the periodontal fibers and their attachment in rat molar periodontium incident to orthodontic tooth movement. Scand J. Dent. Res. 81:467-80, 1973 .

33. Rygh, P. and Selvig, K.A. Erythrocytic crystallization in rat molar periodontium incident to orthodontic tooth movement. Scand. J. Dent. Res. 81:62-73, 1973 .

34. Kvam, E. Scanning electron microscopy of tissue changes on the pressure surface of human premolars following tooth movement. Scand. J. Dent. Res. 80:357-68, 1972 .

35. Kvam, E. Scanning electron microscopy of human premolars following experimental tooth movement. Trans. Europ. Orthod. Soc. 1-11, 1972 .

36. Lurie, A.G., Greenberg, R.J. and Kornman, K.S. Subtraction demonstrates crestal bone loss in experimentally induced marginal periodontitis. Oral Med., Oral Surg., Oral Path. 55:537-41, 1983 .

37. Ottolengui, R. The physiological and pathological resorption of tooth roots. Items of Interest. 36:332-62, 1914 .

38. Dellinger, E.L. A histologic and cephalometric investigation of premolar intrusion in the Macaca speciosa monkey. Am. J. Orthod. 53:325-355, 1976.

39. Williams, S. A histomorphometric study of orthodontically induced root resorption. Europ. J. Orthod. 6:35-47, 1984 .

40. Rygh, P. Orthodontic root resorption studied by electron microscopy. Am. J. Orthod. 47:1-16, 1977 .

41. Harstedt, C. and Welander, U. Photographic subtraction. I. Theory of the subtraction image. Acta. Rad. Diag. 16:55964,1975 .

42. Webber, R.L., Ruttiman, U.E. and Grondahl, H.G. X-ray image subtraction as a basis for assessment of periodontal changes. J. Perio. Res. 17:509-11, 1982.

43. Kasle, M.J. and Klein, A.J. Television radiographic evaluation of periapical osseous radiolucencies. Oral surg. $41: 789-96,1976$. 
44. GrondahI, H.G., Grondahl, K. and Webber, R.L. A digital subtraction technique for dental radiography. Oral Surg. 55:96-102, 1983 .

45. Grondahl, H.G., Grondahl, K. and Webber, R.L. Digital subtraction radiography for diagnosis of periodontal bone lesions with simulated high-speed systems. Oral surg. 55:313-18, 1983 .

46. McWilliam, J.S. The application of photographic subtraction in longitudinal cephalometric growth studies. Europ. J. Orthod. 4:29-32, 1982 .

47. Burstone, C.J. Mechanics of the segmented arch technique. Orthod. 39:99-120, 1966 .

48. Selvig, K.A. The fine structure of human cementum. Acta Odont. Scand. 23:423-441, 1965 .

49. Burstone, C.J. and Hanley, K.J. Modern Edgewise Mechanics Segmented Arch Technique. UCONN Syllabus. 